\title{
First Demonstration of Early Warning Gravitational-wave Alerts
}

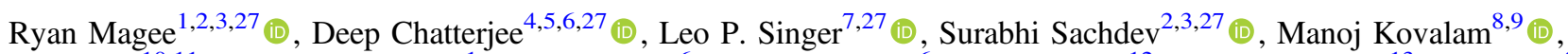
Geoffrey Mo ${ }^{10,11}$ (1), Stuart Anderson ${ }^{1}$, Patrick Brady ${ }^{6}$, Patrick Brockill ${ }^{6}$, Kipp Cannon ${ }^{12}$, Tito Dal Canton ${ }^{13}$ (1) , Qi Chu, Patrick Clearwater $^{8,14}$, Alex Codoreanu ${ }^{8,14}$, Marco Drago ${ }^{15,16}$, Patrick Godwin ${ }^{2,3}$, Shaon Ghosh ${ }^{17}$ (1), Giuseppe Greco ${ }^{18,19}$, Chad Hanna $^{2,3,20}$, Shasvath J. Kapadia ${ }^{21}{ }^{10}$, Erik Katsavounidis ${ }^{10,11}$, Victor Oloworaran ${ }^{8,9}$, Alexander E. Pace ${ }^{2,3}$, Fiona Panther ${ }^{8,9}$, Anwarul Patwary ${ }^{8,9}$, Roberto De Pietri ${ }^{22,23}$, Brandon Piotrzkowski ${ }^{6}$, Tanner Prestegard ${ }^{6}$, Luca Rei ${ }^{24}$, Anala K. Sreekumar ${ }^{8,9}$, Marek J. Szczepańczyk ${ }^{25}$, Vinaya Valsan ${ }^{6}$, Aaron Viets ${ }^{6}$, Madeline Wade ${ }^{26}$, Linqing Wen ${ }^{8,9}$ (i), and John Zweizig ${ }^{1}$

${ }^{1}$ LIGO, California Institute of Technology, Pasadena, CA 91125, USA

${ }^{2}$ Department of Physics, The Pennsylvania State University, University Park, PA 16802, USA

${ }^{3}$ Institute for Gravitation and the Cosmos, The Pennsylvania State University, University Park, PA 16802, USA

${ }^{4}$ Center for Astrophysical Surveys, National Center for Supercomputing Applications, Urbana, IL 61801, USA

${ }^{5}$ Illinois Center for Advanced Studies of the Universe, Department of Physics, University of Illinois at Urbana-Champaign, Urbana, IL 61801, USA

${ }^{6}$ Leonard E. Parker Center for Gravitation, Cosmology, and Astrophysics, University of Wisconsin-Milwaukee, Milwaukee, WI 53201, USA

Astroparticle Physics Laboratory, NASA Goddard Space Flight Center, Mail Code 661, Greenbelt, MD 20771, USA

${ }^{8}$ Australian Research Council Centre of Excellence for Gravitational Wave Discovery (OzGrav), Australia

${ }^{9}$ Department of Physics, University of Western Australia, Crawley WA 6009, Australia

${ }^{10}$ LIGO Laboratory, Massachusetts Institute of Technology, Cambridge, MA 02139, USA

${ }^{11}$ Department of Physics and Kavli Institute for Astrophysics and Space Research, Massachusetts Institute of Technology, Cambridge, MA 02139, USA

${ }^{12}$ Research Center for the Early Universe, The University of Tokyo, 113-0033, Japan

${ }^{13}$ Université Paris-Saclay, CNRS/IN2P3, IJCLab, F-91405 Orsay, France

${ }^{14}$ Gravitational Wave Data Centre, Swinburne University, Hawthorn VIC 3122, Australia

${ }^{15}$ Università di Roma "La Sapienza," I-00185 Roma, Italy

${ }^{16}$ INFN, Sezione di Roma, I-00185 Roma, Italy

${ }^{17}$ Department of Physics and Astronomy, Montclair State University, Montclair, NJ 07043, USA

${ }_{18}^{18}$ Università degli Studi di Urbino "Carlo Bo", I-61029 Urbino, Italy

${ }^{19}$ INFN, Sezione di Firenze, I-50019 Sesto Fiorentino, Firenze, Italy

${ }^{20}$ Department of Astronomy and Astrophysics, The Pennsylvania State University, University Park, PA 16802, USA

${ }^{21}$ International Centre for Theoretical Sciences, Tata Institute of Fundamental Research, Bangalore 560089, India

${ }_{22}$ Dipartimento di Scienze Matematiche, Fisiche e Informatiche, Università di Parma, I-43124 Parma, Italy

23 7INFN, Sezione di Milano Bicocca, Gruppo Collegato di Parma, I-43124 Parma, Italy

${ }^{24}$ INFN, Sezione di Genova, I-16146 Genova, Italy

${ }^{25}$ University of Florida, Gainesville, FL 32611, USA

${ }^{26}$ Department of Physics, Kenyon College, Gambier, OH 43022, USA

Received 2021 February 25; accepted 2021 March 10; published 2021 April 6

\begin{abstract}
Gravitational-wave observations became commonplace in Advanced LIGO-Virgo's recently concluded third observing run. 56 nonretracted candidates were identified and publicly announced in near real time. Gravitational waves from binary neutron star mergers, however, remain of special interest since they can be precursors to highenergy astrophysical phenomena like $\gamma$-ray bursts and kilonovae. While late-time electromagnetic emissions provide important information about the astrophysical processes within, the prompt emission along with gravitational waves uniquely reveals the extreme matter and gravity during - and in the seconds followingmerger. Rapid communication of source location and properties from the gravitational-wave data is crucial to facilitate multimessenger follow-up of such sources. This is especially enabled if the partner facilities are forewarned via an early warning (pre-merger) alert. Here we describe the commissioning and performance of such a low-latency infrastructure within LIGO-Virgo. We present results from an end-to-end mock data challenge that detects binary neutron star mergers and alerts partner facilities before merger. We set expectations for these alerts in future observing runs.
\end{abstract}

Unified Astronomy Thesaurus concepts: Gravitational waves (678); Gravitational wave astronomy (675); Neutron stars (1108); High energy astrophysics (739)

\section{Introduction}

The field of gravitational-wave astronomy has exploded in the years following the first direct observation of gravitational waves (GWs) from a binary black hole (BBH) merger (Abbott et al. 2016). Since then, LIGO-Virgo have published 49 candidate events, many of which were identified in low-latency; ${ }^{28}$ these include two binary neutron star (BNS) and two neutron

\footnotetext{
27 These authors contributed equally to this work.

28 Some of the 56 have not yet appeared in a LIGO-Virgo publication.
}

star-black hole (NSBH) candidates (Abbott et al. 2020a). The detection of GWs from compact binaries, especially from BBHs, has become routine. GWs from BNS and NSBH mergers, however, remain rare. BNS and NSBH mergers are of special interest due to the possibility of counterpart electromagnetic (EM) signals. For BNS mergers, in particular, it has long been hypothesized that the central engine (post merger) can launch short gamma-ray bursts (SGRBs; Lattimer \& Schramm 1976; Lee \& Ramirez-Ruiz 2007), kilonovae (Li \& Paczynski 1998; Metzger et al. 2010), and radio waves and X-rays post merger (Nakar \& Piran 2011; Metzger \& Berger 2012). In the special case of the 
presence of a magnetized NS, it can also lead to GRB precursors before the merger (Metzger \& Zivancev 2016).

Although the improvement in Advanced LIGO-Virgo's sensitivity was paralleled by analogous advancements in the field of time-domain astronomy, the first observed BNS merger, GW170817 (Abbott et al. 2017c), remains the only realization of multimessenger astronomy (MMA) with GWs. The coincident observation of GWs followed by an SGRB, GRB 170817A, and the kilonova AT 2017gfo, (Abbott et al. 2017d) bore evidence to the several-decade-old hypothesis that compact object mergers were progenitors of these exotic transients. The joint observations also contributed greatly to our understanding of fundamental physics (Abbott et al. 2017b, 2019b) and astrophysical processes associated with extreme environments (Abbott et al. 2017a; Nicholl et al. 2017). Despite the plethora of late-time observations made starting $\sim 8 \mathrm{hr}$ after coalescence (Abbott et al. 2017d), observations of the prompt spectra were precluded by nonstationarities in the LIGO-Livingston interferometer and delays in Virgo data transfer. The alert and sky localization were distributed to partner observatories $\sim 40$ minutes (LIGO Scientific Collaboration 2017a) and $\sim 5 \mathrm{hr}$ (LIGO Scientific Collaboration 2017b), respectively, after the signal arrived at the detectors; by this time, the source had set below the horizon for northern hemisphere telescopes. The circumstances surrounding this delay were unusual, but it is crucial for LIGO-Virgo to distribute alerts as quickly as possible to maximize the chance of additional multimessenger observations.

The serendipitous discovery of GRB 170717A by Fermi and INTEGRAL show the importance of catching the prompt EM emission to our understanding of merging compact binaries. EM observatories have begun to develop capacity to perform targeted observations in response to preliminary Gamma-ray Coordinates Network (GCN) notices produced by pre-merger detections. For example, the Murchison Wide-Field Array (MWA) radio telescope has a large field of view ideally suited to searching for precursor and prompt radio emission from $\mathrm{GW}$ sources and an established observing plan to respond to pre-merger detections (James et al. 2019). Swift-BAT has recently also demonstrated the potential to respond autonomously to extremely low-latency triggers in the future, with the introduction of an on-board subthreshold trigger recovery algorithm (GUANO; Tohuvavohu et al. 2020). By the beginning of Advanced LIGO-Virgo's fourth observing run (O4), it is expected that established missions and observatories will be joined by next generation facilities like the Rubin Observatory (Ivezić et al. 2019). This greatly improves the chances of performing targeted follow-up observations of prompt, or even precursor (Troja et al. 2010; Tsang et al. 2012), emission from compact binary mergers provided that pre-merger alerts can be issued.

LIGO-Virgo has since streamlined the alert process (see Figure 3). Advanced LIGO saw the dawn of autonomously distributed Preliminary Advanced Virgo's Notices (LIGO Scientific Collaboration 2019), ${ }^{29}$ which allowed LIGO-Virgo to notify the world of candidate signals within $7.0_{-4}^{+92}$ minutes ${ }^{30}$ of observation. To further enable EM-GW observations, we can leverage the long-lived nature of BNSs in the sensitive band of advanced ground-based $\mathrm{GW}$ detectors to make pre-merger detections (Cannon et al. 2012; Chu et al. 2016). This was recently demonstrated by Sachdev et al. (2020) and Nitz et al. (2020). The

\footnotetext{
29 https://gcn.gsfc.nasa.gov/

30 The $95 \%$ reported here is severely impacted by several high latency events that evaded automated procedures.
}

early detection and communication of GWs from BNSs aims to facilitate EM follow-up efforts by further reducing the latency of alerts and improving prospects of capturing the initial spectra.

In this Letter we describe the commissioning and performance of the low-latency subsystem within Advanced LIGO-Virgo that is able to provide pre-merger alerts for electromagnetically bright compact binaries. We begin by describing the end-to-end lowlatency workflow in Section 2, from the time of data acquisition to the dissemination of public alerts. We then assess the performance of a subset of this infrastructure in a mock data challenge described in Section 3, with special emphasis placed on premerger alerts. We demonstrate that Preliminary GCN Notices can be distributed with true negative latencies: partner observatories receive sky localizations and source information before the binary has completed its merger. We report on the improved latencies at each step of the workflow, and set expectations for pre-merger alerts in $\mathrm{O} 4$ and next generation detectors in Section 4.

\section{Analysis}

The low-latency workflow begins with data acquisition at each interferometer. The digital signal from the output photodiode is initially calibrated by a pipeline that runs on the set of computers that directly control the interferometer. The calibrated data, while produced with near-zero latency, are not yet accurate enough for use by low-latency gravitational-wave searches. The data are broadcast to a set of computers where a GStreamer-based pipeline corrects the strain data to achieve the required level of accuracy (Viets et al. 2018). This pipeline writes the calibrated strain data to a proprietary LIGO frame data format and then transfers them to computing sites. There, the calibrated data are ingested by the complete set of low-latency full bandwidth GW pipelines: cWB (Klimenko \& Mitselmakher 2004; Klimenko et al. 2005, 2006, 2011, 2016), GstLAL (Messick et al. 2017; Sachdev et al. 2019; Hanna et al. 2020), MBTAOnline (Adams et al. 2015), PyCBC Live (Nitz et al. 2018; Dal Canton et al. 2020), and SPIIR (Hooper et al. 2012; Luan et al. 2012; Liu et al. 2012; Chu 2017; Guo et al. 2018). For the first time, we also incorporate two matched-filter-based pipelines focused on pre-merger detection into our workflow: GstLAL (Cannon et al. 2012; Sachdev et al. 2020) and SPIIR (Chu et al. 2020). All detection pipelines analyze the data for GWs and assign significances to candidate triggers. Candidates that are assigned false alarm rates (FARs) less than one per hour ${ }^{31}$ are uploaded to the GRAvitational-wave Candidate Event DataBase (GraceDB) ${ }^{32}$ alongside data required downstream in the alert process.

After candidates are uploaded, the task manager GWCelery ${ }^{33}$ interacts with low-latency searches and GraceDB to orchestrate a number of parallel and interconnected processes which, in the event of a discovery, culminates in the dissemination of GCN Notices. GWCelery provided the semiautomated infrastructure for public alerts in $\mathrm{O} 3$, as well as for the mock data challenge reported here. The major subsystems include:

1. The listener for LVAlert, which is a publish-subscribe system used by GraceDB to push machine-readable notifications about its state.

2. The Superevent Manager, which clusters and merges related candidates into superevents. ${ }^{34}$

\footnotetext{
31 The no trials factor is applied to the candidate upload threshold.

32 https://gracedb.ligo.org/

33 https://gwcelery.readthedocs.io/

${ }^{34}$ https://emfollow.docs.ligo.org/userguide/analysis/superevents.html
} 
3. The client functionality to interact with GraceDB. ${ }^{35}$

4. The GCN listener that listens for notices from external facilities to spot coincidences with GW candidates.

5. The External Trigger Manager, which correlates gravitational-wave events with GRB, neutrino, and supernova events.

6. The GCN broker that disseminates GW candidate information for external consumption.

7. The Orchestrator, which executes the per-(super)event annotation workflow.

After candidate events are uploaded by detection pipelines, they are localized via BAYESTAR (Singer \& Price 2016), given a probability of having an electromagnetic counterpart (Chatterjee et al. 2020), and assigned a source-category based astrophysical probability under the assumption that astrophysical and terrestrial triggers occur as independent Poisson processes (Kapadia et al. 2020). Events are checked for temporal and, when possible, spatial coincidences with gamma-ray bursts or neutrino bursts using the RAVEN pipeline (Urban 2016). A joint significance is calculated to decide whether the joint candidate should be published.

BAYESTAR was optimized in order to support early warning localizations which led to a median run time of $0.5 \mathrm{~s}$ per event for early warning triggers and $1.1 \mathrm{~s}$ per event for full bandwidth triggers. The latter is a $4.2 \times$ speedup compared to usual $\mathrm{O} 3$ performance. The significant changes included rearrangement of loops to improve memory access patterns and make better use of $\times 86$ 64 vector instructions, changes to the input data handling to distinguish properly between the merger time and the cutoff time of early warning templates, and the redesign of the reconstruction filter that is used to sample the SNR time series for likelihood evaluation to use a lower sample rate. ${ }^{36}$

To mitigate the effect of noise transients, basic data quality checks are also performed for every candidate uploaded to GraceDB. In particular, specific state vectors are checked to ensure that candidate events occur during times when the relevant detectors are in observing mode and to verify that there are no coincident hardware injections.

A qualitative overview of the entire pipeline and the various (sub)systems mentioned above is illustrated in Figure 1. A heuristic waveform evolution and the effect of different early warning template cutoff times on the accumulated signal-tonoise ratio $(\mathrm{S} / \mathrm{N})$ and the sky localization is also shown.

\section{Results}

To demonstrate the robustness of the alert infrastructure, we describe the results of a mock data challenge carried out between 2020 June 111700 UTC and 2020 June 191700 UTC. Data previously collected during $\mathrm{O} 3$ were replayed as a mock low-latency analysis. We note that since the challenge relied on previously collected data, it was impossible to test the full lowlatency workflow; notably, data transfer and calibration latencies are not included $(\sim 5 \mathrm{~s})$. The test therefore begins with the detection pipelines, but otherwise follows a workflow identical to Advanced LIGO-Virgo observing runs.

The FAR threshold set for issuing early warning test notices was chosen to be 1 per day. Full bandwidth triggers used the

\footnotetext{
35 https://gracedb-sdk.readthedocs.io

36 The early warning templates are Nyquist critically sampled which could lead to ringing artifacts.
}

same FAR threshold set throughout $\mathrm{O} 3$ for public alerts (one per two months). ${ }^{37}$ At fixed FAR, the astrophysical probability (Kapadia et al. 2020) associated with pre-merger analyses is lower than for full bandwidth analyses. Due to this fact, combined with our chosen higher FAR threshold for early warning alerts, we issued retraction circulars for early warning candidates that were not also identified by the full bandwidth analyses. There were no retraction criteria set for full bandwidth triggers.

During the mock data challenge, eight candidates were published via the test GCN. Three candidates were identified by only the full bandwidth analyses and were distributed via notice and circular(LIGO Scientific Collaboration 2020a, 2020b, 2020c). The remaining five public candidates were identified only by the early warning pipelines and were distributed via GCN notices to subscribers of test alerts. None of these five candidates were observed in the full bandwidth analyses; they were therefore subsequently retracted(LIGO Scientific Collaboration 2020d, 2020e, 2020f, 2020g, 2020h). Out of the five retracted triggers, four came from the GstLAL early warning pipeline, while one was issued by the SPIIR early warning pipeline. An authentication issue prevented the SPIIR pipeline from issuing additional events past the FAR threshold. A summary of the five early warning alerts is given in Table 1 in the Appendix.

Although only five pre-merger candidates passed the early warning public alert threshold, GstLAL and SPIIR uploaded 82 and 141 early warning candidate events, respectively, to GraceDB. We use the metadata associated with these uploads to produce Figure 2. From the events crossing threshold we see that the maximum delivery time from event upload is $15 \mathrm{~s}$, independent of pipeline. This enables $\sim 85 \%$ and $\sim 35 \%$ of the GstLAL and SPIIR candidates, respectively, to be localized before merger.

\section{Looking Ahead}

Early warning alerts using real data have not yet been released by the LIGO-Virgo collaboration. Despite the steady improvement of the alert infrastructure (Figure 3), there remain several areas for improvement in the processing of data and production of alerts if the collaboration decides to pursue premerger triggers. As previously mentioned, low-latency data calibration is currently a two step process; the near-zero-latency pipeline is corrected by a secondary GStreamer-based pipeline. Work is underway to reduce this to a single calibration step to reduce latency by $\mathcal{O}$ (seconds). The calibrated data are transferred from the detector sites to the computing clusters in $\sim 4 \mathrm{~s}$, and afterward at the cluster level using Kafka, ${ }^{38}$ with an additional $\sim 0.1 \mathrm{~s}$. Another $1 \mathrm{~s}$ of latency ${ }^{39}$ is attributed to the choice to distribute data via frame files. A number of improvements are under development to reduce this latency budget.

Reductions to the noise budget at frequencies $\lesssim 30 \mathrm{~Hz}$ will improve the possibility of detection pipelines identifying signals long before merger. We estimate that if the noise floor below $30 \mathrm{~Hz}$ remains unchanged from $\mathrm{O} 3$, the recovered $\mathrm{S} / \mathrm{N} 1$ minute and 30 s before merger will be $\sim 50 \%$ and $\sim 20 \%$ less, respectively, than if the detectors reach the previously projected

\footnotetext{
37 A trials factor is applied on top of this threshold to account for the two early warning and four full bandwidth matched filter pipelines.

38 https://kafka.apache.org/

39 Four seconds for Virgo data.
} 


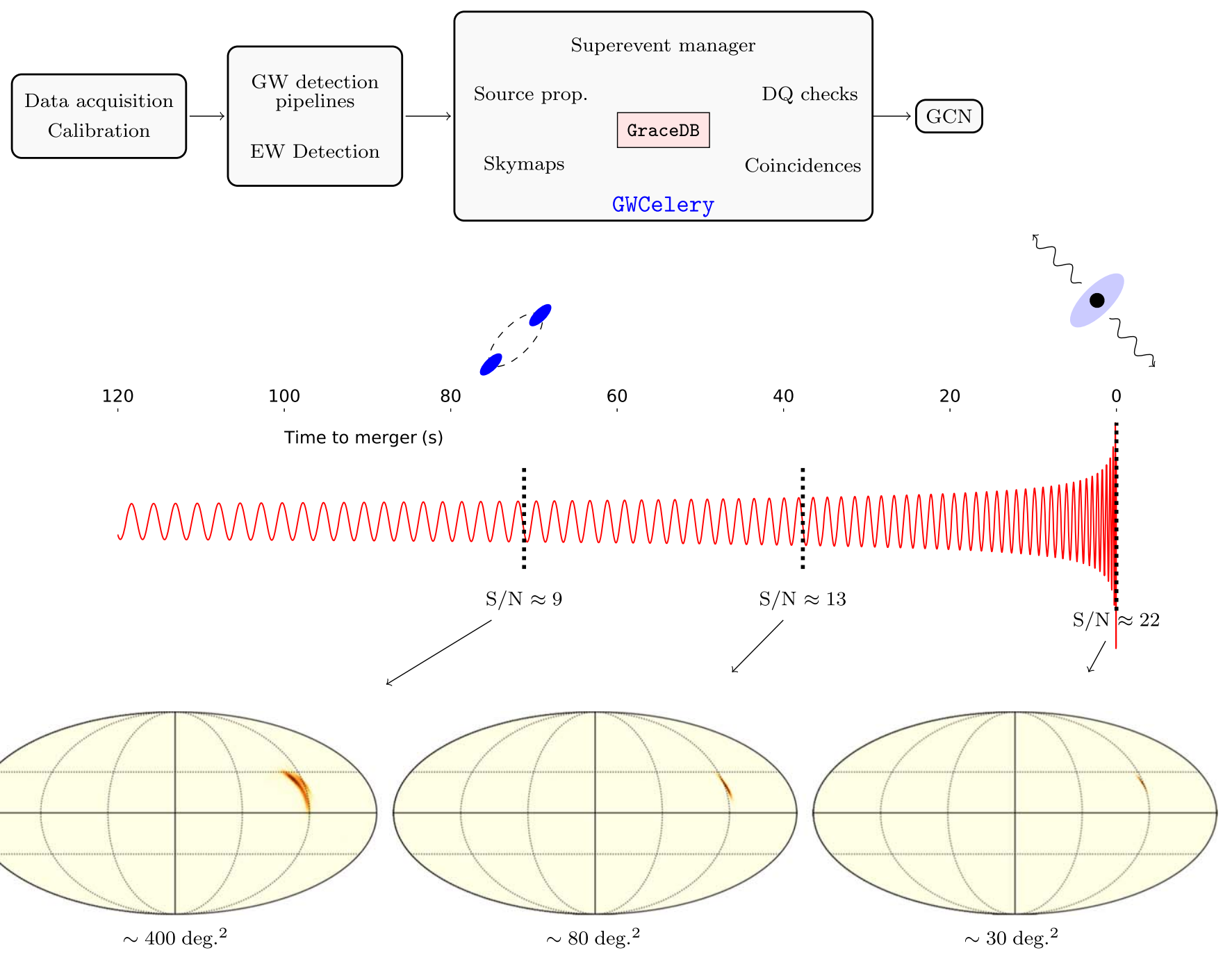

Figure 1. The upper half of the figure illustrates the complete pipeline and interaction of the various (sub)systems, mentioned in Section 2, responsible for disseminating early warning alerts. The waveform evolution with time is shown in the bottom half along with the dependence of the sky-localization area on the cutoff time of the early warning templates and the accumulated $\mathrm{S} / \mathrm{N}$ during the binary inspiral. The waveforms, time to merger, $\mathrm{S} / \mathrm{N}$, and localizations in this figure are qualitative.

O4 sensitivity. The effect is less severe for early warning times just before merger, but low frequency noise is a major barrier to advancing alerts.

Figures 2 and 3 demonstrate that the GW alert system is capable of providing GW alerts before merger, but they do not consider the prospects for detection from an astrophysical source population. We generate a population of simulated BNS signals, henceforth referred to as injections, using the TaylorF2 (Sathyaprakash \& Dhurandhar 1991; Blanchet et al. 1995, 2005; Buonanno et al. 2009) waveform model. Both source-frame component masses are drawn from a Gaussian distribution between $1.0 M_{\odot}<m_{1}$, $m_{2}<2.0 M_{\odot}$ with mean mass of $1.33 M_{\odot}$ and standard deviation of $0.09 M_{\odot}$, modeled after observations of galactic BNSs (Özel \& Freire 2016). ${ }^{40}$ The neutron stars in the population are nonspinning, motivated by the low spins of BNSs expected to merge within a Hubble time (Burgay et al. 2003; Zhu et al. 2018). The signals are distributed uniformly in comoving volume up to a redshift of $z=0.2$. We consider a network of

\footnotetext{
$\overline{40}$ Note that if GW190425 is a BNS, then galactic measurements are not representative of neutron star masses.
}

four GW detectors: LIGO-Hanford, LIGO-Livingston, Virgo, and KAGRA at their projected O4 sensitivities. ${ }^{41}$ We simulate the results of an early warning matched-filtering pipeline by considering six different discrete frequency cutoffs: $29,32,38$, 49,56 , and $1024 \mathrm{~Hz}$ to analyze signal recovery at (approximately) 58, 44, 28, 14, 10, and $0 \mathrm{~s}$ before merger, motivated by Sachdev et al. (2020). We calculate the network S/N of each injection at each frequency cutoff and consider the events that pass an S/N cutoff of 12.0 as "detected." We then calculate the sky posteriors for each of the detected signals by using BAYESTAR (Singer \& Price 2016). We use the most recent BNS local merger rate from Abbott et al. (2020b) of $320_{-240}^{+410} \mathrm{Gpc}^{-3} \mathrm{yr}^{-1}$ to estimate the number of events detected per year in the detector network. In Figure 4(a) we see that our optimistic scenario predicts $5_{-4}^{+7}$ GCN will be received $1 \mathrm{~s}$ before merger per year, while our pessimistic scenario predicts $\mathcal{O}$ (1) GCN will be received $1 \mathrm{~s}$ before merger per year considering the higher end of the BNS rate. Figure 4(b) predicts that $\sim 9$ events will be detected per year, out of which $\sim 20 \%$

\footnotetext{
$\overline{{ }^{41} \text { https://dcc.ligo.org/LIGO-T2000012/public }}$
} 

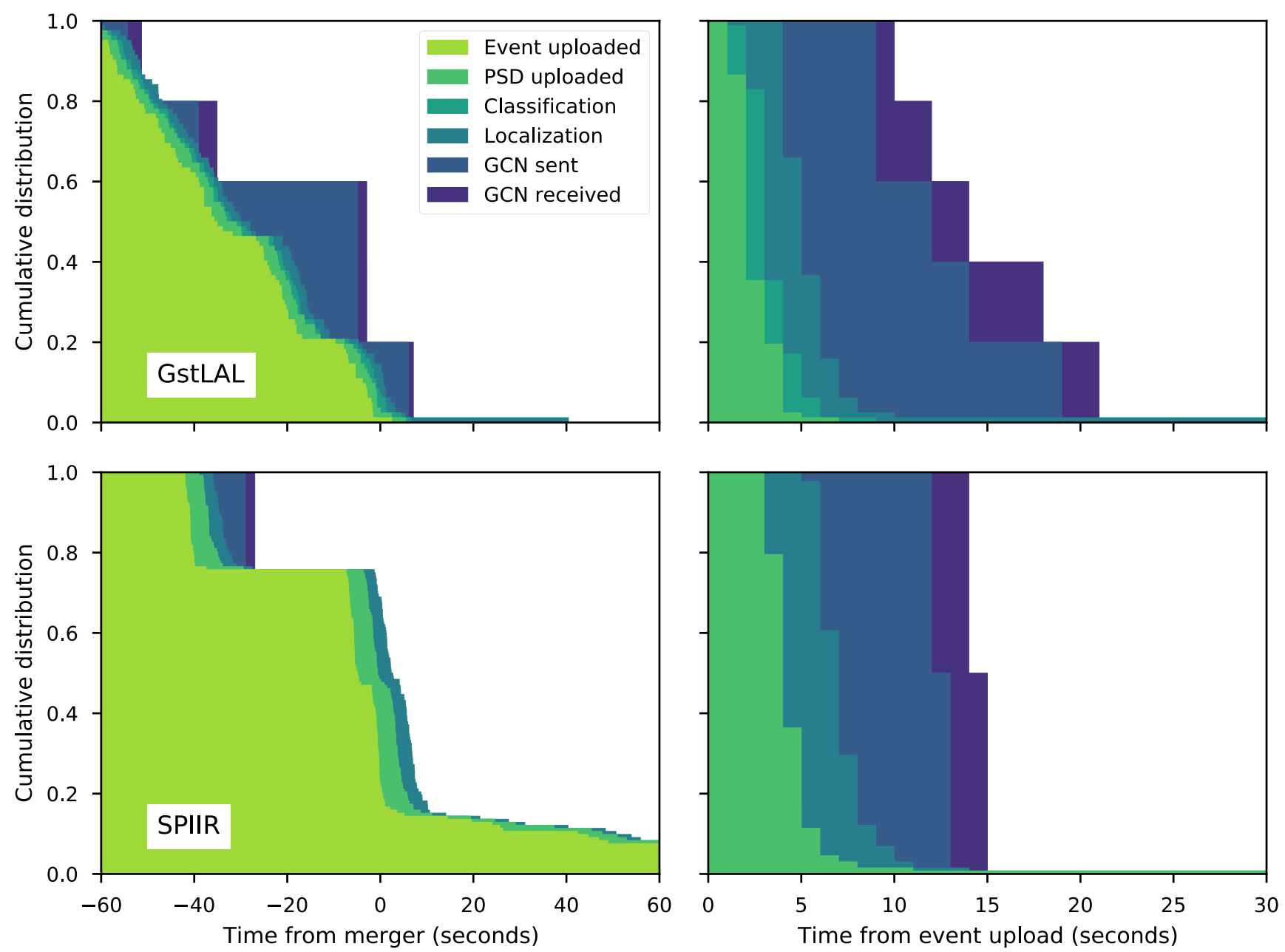

Figure 2. Latencies associated with early warning uploads from the GstLAL (top) and SPIIR (bottom) pipelines. Design differences between the pipelines lead to distinct distributions for the time before merger at which a candidate is identified. The left panels indicate that $\sim 85 \%$ and $~ 35 \%$ of the uploaded GstLAL and SPIIR candidates, respectively, are localized prior to merger. The right panels demonstrate that despite differences in latencies associated with event identification, the scatter of the remaining processes is remarkably similar.

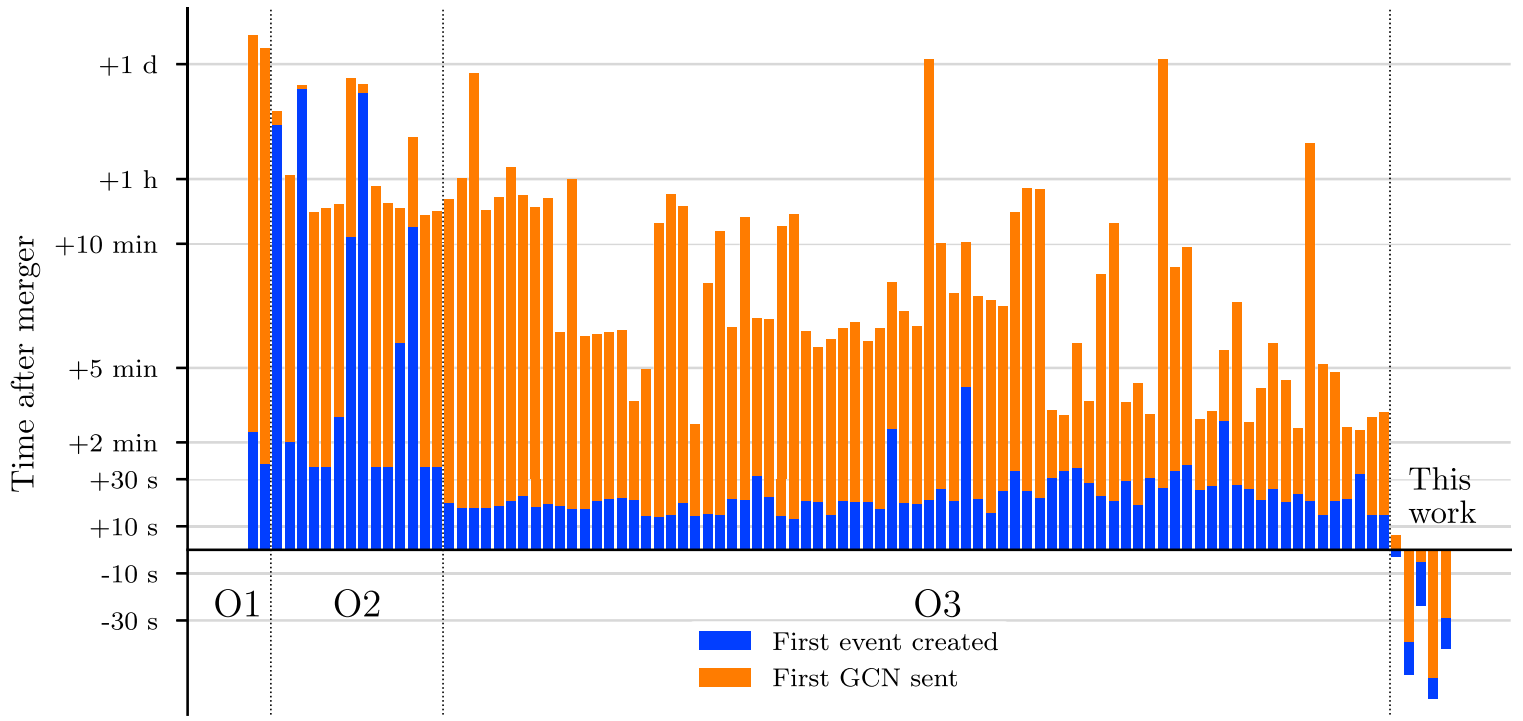

Figure 3. A history of end-to-end latencies across public alerts in the first three observing runs and the mock data challenge is presented here (Abbott et al. 2019a).

$(\sim 1.3 \%)$ will be detected $10 \mathrm{~s}(60 \mathrm{~s})$ before merger. Further, $\sim 3 \%$ of the detectable events ( 1 BNS every 3-4 yr) will be detected $10 \mathrm{~s}$ prior to merger and have a localization less than
$100 \mathrm{deg}^{2}$ at $\mathrm{O} 4$ sensitivities. This highlights the need for continued latency improvements in advance of $\mathrm{O} 4$ to maximize the potential of capturing prompt emission. 


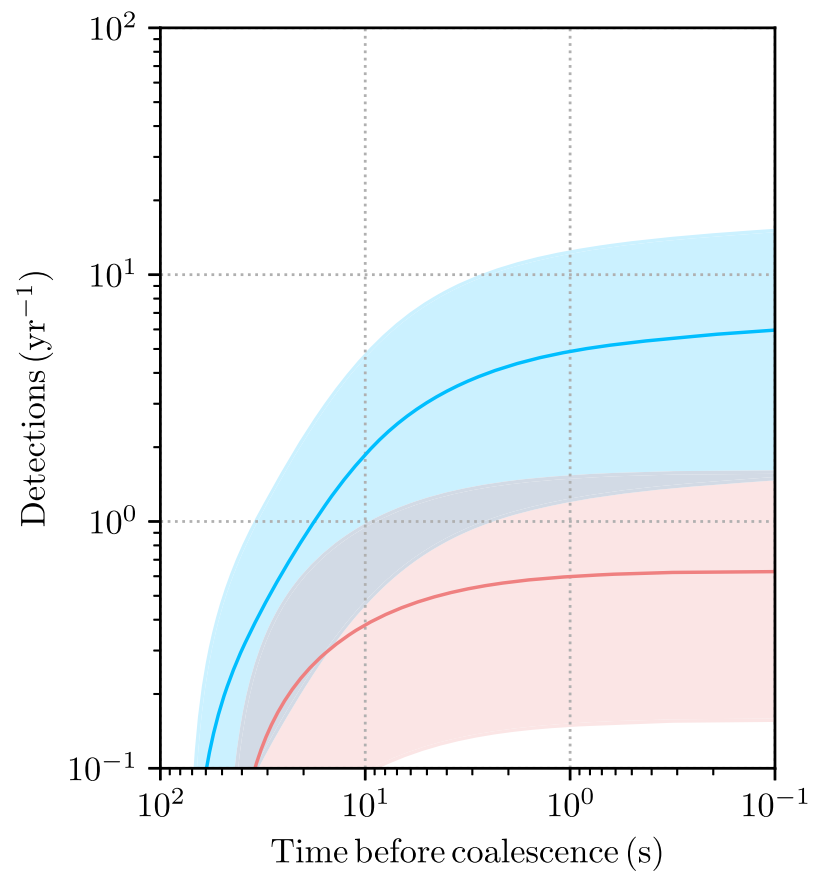

(a)

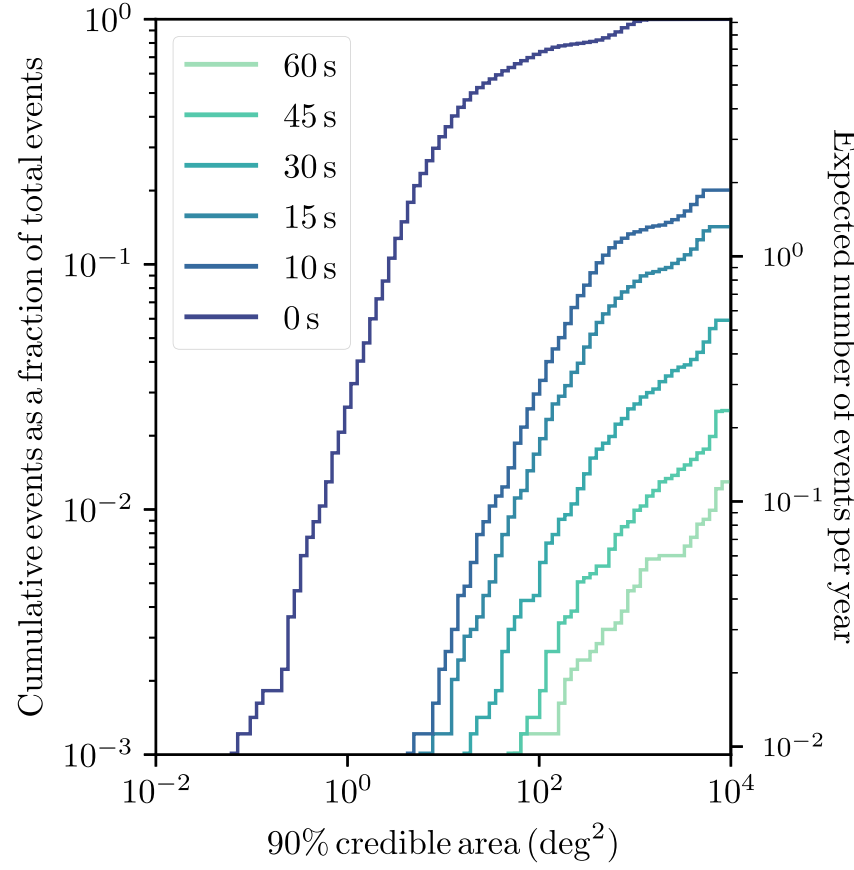

(b)

Figure 4. (a) Projected $\mathrm{O} 4$ early warning detection rate assuming $0 \mathrm{~s}$ (blue) and $25 \mathrm{~s}$ (red) end-to-end latencies from the GW alert system. The worst case scenario assumes $5 \mathrm{~s}$ for calibration and data transfer, $5 \mathrm{~s}$ for pipeline analysis, and $15 \mathrm{~s}$ for event upload and GCN creation. The rate of expected detections was estimated from a simulated data set assuming a 100\% detector duty cycle for the 4-detector HLVK network. The uncertainty bands reflect the (5\%, 95\%) confidence region for the BNS rate. Signals with network S/Ns greater than 12 are considered recovered. (b) The expected localization distribution for BNS detections at six approximate early warning times. No latencies are included in this figure. The inclusion of an end-to-end latency does not shift the histogram itself; the labeled times before merger would all systematically shift instead. Both plots use the BNS rates estimated in Abbott et al. (2020b).

In the design sensitivity era with three detectors, Sachdev et al. (2020) have shown that about half of the total detectable BNSs will be found $10 \mathrm{~s}$ before merger, and about $2 \%$ will be identified before merger and localized to within $100 \mathrm{deg}^{2}$. Sachdev et al. (2020) used the GstLAL pipeline in an early warning configuration to assign FARs to simulated BNS signals to estimate these rates. ${ }^{42}$ We extend this to include KAGRA in the detector network, but we estimate rates based on a fiducial $\mathrm{S} / \mathrm{N}$ cutoff of 12 . We find that our zero-latency scenario improves to $\sim 2$ BNS observable 1 minute before coalescence. Assuming $25 \mathrm{~s}$ of pipeline latency, $\sim 1$ BNS will be localized and disseminated 1 minute before merger every 2 yr. The localization prospects similarly improve. At design sensitivity, $\sim 3$ BNS every year will be detected $10 \mathrm{~s}$ prior to merger and have localizations $\lesssim 100 \mathrm{deg}^{2}, \sim 2$ signals per year will be detected $15 \mathrm{~s}$ prior to merger with similar localization. The detection rates estimated by Nitz et al. (2020) are comparable to ours, considering their use of a larger BNS rate density ( $\sim 3$ times ours) and a less strict criterion for the detectability of a signal (network $\mathrm{S} / \mathrm{N}>10$ ).

The next generation of ground-based interferometers will offer unparalleled early warning capabilities. Using a similar $\mathrm{S} / \mathrm{N}$ detection threshold (but further mandating that at least two interferometers measure S/Ns above 5.5), Chan et al. (2018) found that the Einstein Telescope can alert observers up to 20 $\mathrm{hr}$ in advance for $58 \%$ of detectable BNS at $200 \mathrm{Mpc}$ and $100 \%$ at $40 \mathrm{Mpc}$. The majority of these signals will be well localized.

\footnotetext{
42 Note that the estimated BNS rate at the time of Sachdev et al. (2020) was approximately three times larger than the updated rate presented in Abbott et al. (2020b).
}

A similar study by Akcay (2019) with an S/N detection threshold of 15 found that the Einstein Telescope will provide early notice for $\mathcal{O}\left(10^{2}\right)$ BNS mergers in the next decade.

We are grateful to B.S. Sathyaprakash for reviewing our manuscript and providing useful comments. We thank the LIGO Laboratory for use of its computing facility to make this work possible, and we gratefully acknowledge the support of the National Science Foundation (NSF) grants PHY-0757058 and PHY-0823459. C.H. gratefully acknowledges the support of NSF grant OAC-1841480. D.C. acknowledges NSF grant No. PHY1700765 and PHY-1912649, and is supported by the Illinois Survey Science Fellowship of the Center for Astrophysical Surveys (CAPS) at the University of Illinois Urbana-Champaign. S.S. is supported by the Eberly Research Funds of Penn State, The Pennsylvania State University, University Park, Pennsylvania. G.M. is supported by the NSF through award PHY-1764464 to the LIGO Laboratory. M.K., Q.C., F.P., L.W., A.P., A.S., and V. O. acknowledge the funding from Australian Research Council (ARC) Centre of Excellence for Gravitational Wave Discovery OzGrav under grant CE170100004.

Facilities: LIGO, EGO:Virgo.

Software: astropy (Astropy Collaboration et al. 2013), numpy (Harris et al. 2020), matplotlib (Hunter 2007), iPython (Perez \& Granger 2007), pandas (McKinney 2010), gwpy (Macleod et al. 2020), celery (Solem 2020).

\section{Appendix}

A summary of the five early warning alerts described in Section 3 are included here in Table 1. 
Table 1

A Summary of the Five Early Warning Alert Information and Latencies from the Mock Data Challenge Described in Section 3

\begin{tabular}{|c|c|c|c|c|c|c|c|}
\hline \multirow{2}{*}{ Superevent } & \multirow{2}{*}{ Date (UTC) } & \multirow{2}{*}{ FAR } & \multicolumn{4}{|c|}{ Latency } & \multirow{2}{*}{ GCNs } \\
\hline & & & Event & Superevent & Skymap & Notice & \\
\hline MS200615h & 2020-06-15 00:35:40 & $2.02 \mathrm{e}-06$ & -2.9 & -1.9 & 0.1 & 7.1 & https://gcn.gsfc.nasa.gov/gcn3/27951.gcn3 \\
\hline MS200618aq & 2020-06-18 05:47:05 & $1.78 \mathrm{e}-07$ & -53.1 & -52.1 & -50.1 & -35.1 & https://gcn.gsfc.nasa.gov/gen3/27990.gen3 \\
\hline MS200618bq & 2020-06-18 11:00:59 & $3.50 \mathrm{e}-06$ & -16.9 & -21.9 & -11.9 & -2.9 & https://gcn.gsfc.nasa.gov/gcn3/27987.gcn3 \\
\hline MS200618bx & 2020-06-18 12:17:08 & $3.76 \mathrm{e}-06$ & -63.3 & -62.3 & -59.3 & -51.3 & https://gcn.gsfc.nasa.gov/gcn3/27988.gcn3 \\
\hline MS200619bf & 2020-06-19 10:24:43 & $1.91 \mathrm{e}-06$ & -41.0 & -40.0 & -35.0 & -27.0 & https://gcn.gsfc.nasa.gov/gcn3/27989.gcn3 \\
\hline
\end{tabular}

Note. Among the five, MS200619bf was reported by the SPIIR pipeline, while the others were reported from GstLAL. The latencies are broken down in steps of the event being uploaded into GraceDB, the superevent being created, the skymap being available for the preferred event, and the notice being acknowledged by GCN.

\section{ORCID iDs}

Ryan Magee (i) https://orcid.org/0000-0001-9769-531X Deep Chatterjee (i) https://orcid.org/0000-0003-0038-5468 Leo P. Singer (D) https://orcid.org/0000-0001-9898-5597 Surabhi Sachdev (i) https://orcid.org/0000-0002-0525-2317 Manoj Kovalam (i) https://orcid.org/0000-0001-8143-9696 Geoffrey Mo (iD https://orcid.org/0000-0001-6331-112X Tito Dal Canton (i) https://orcid.org/0000-0001-5078-9044 Shaon Ghosh (iD https://orcid.org/0000-0003-4259-8592 Shasvath J. Kapadia (10 https://orcid.org/0000-0001$5318-1253$

Linqing Wen (ib https://orcid.org/0000-0001-7987-295X

\section{References}

Abbott, B. P., Abbott, R., Abbott, T. D., et al. 2016, PhRvL, 116, 061102 Abbott, B. P., Abbott, R., Abbott, T. D., et al. 2017a, ApJL, 850, L39 Abbott, B. P., Abbott, R., Abbott, T. D., et al. 2017b, ApJL, 848, L13 Abbott, B. P., Abbott, R., Abbott, T. D., et al. 2017c, PhRvL, 119, 161101 Abbott, B. P., Abbott, R., Abbott, T. D., et al. 2017d, ApJL, 848, L12 Abbott, B. P., Abbott, R., Abbott, T. D., et al. 2019a, ApJ, 875, 161 Abbott, B. P., Abbott, R., Abbott, T. D., et al. 2019b, PhRvD, 100, 104036 Abbott, R., Abbott, T. D., Abraham, S., et al. 2020a, arXiv:2010.14527 Abbott, R., Abbott, T. D., Abraham, S., et al. 2020b, arXiv:2010.14533 Adams, T., Buskulic, D., Germain, V., et al. 2015, CQGra, 33, 175012 Akcay, S. 2019, AnP, 531, 1800365

Astropy Collaboration, Robitaille, T. P., Tollerud, E. J., et al. 2013, A\&A, 558, A33

Blanchet, L., Damour, T., Esposito-Farese, G., \& Iyer, B. R. 2005, PhRvD, 71, 124004

Blanchet, L., Damour, T., Iyer, B. R., Will, C. M., \& Wiseman, A. 1995, PhRvL, 74, 3515

Buonanno, A., Iyer, B., Ochsner, E., Pan, Y., \& Sathyaprakash, B. S. 2009, PhRvD, 80, 084043

Burgay, M., D'Amico, N., Possenti, A., et al. 2003, Natur, 426, 531

Cannon, K., Cariou, R., Chapman, A., et al. 2012, ApJ, 748, 136

Chan, M. L., Messenger, C., Heng, I. S., \& Hendry, M. 2018, PhRvD, 97, 123014

Chatterjee, D., Ghosh, S., Brady, P. R., et al. 2020, ApJ, 896, 54

Chu, Q. 2017, PhD thesis, The University of Western Australia, doi:10.4225/ 23/5987feb0a789c

Chu, Q., Howell, E. J., Rowlinson, A., et al. 2016, MNRAS, 459, 121

Chu, Q., Kovalam, M., Wen, L., et al. 2020, arXiv:2011.06787

Dal Canton, T., Nitz, A. H., Gadre, B., et al. 2020, arXiv:2008.07494

Guo, X., Chu, Q., Chung, S. K., et al. 2018, CoPhC, 231, 62

Hanna, C., Caudill, S., Messick, C., et al. 2020, PhRvD, 101, 022003

Harris, C. R., Millman, K. J., van der Walt, S. J., et al. 2020, Natur, 585, 357

Hooper, S., Chung, S. K., Luan, J., et al. 2012, PhRvD, 86, 024012

Hunter, J. D. 2007, CSE, 9, 90
Ivezić, V. Z., Kahn, S. M., Tyson, J. A., et al. 2019, ApJ, 873, 111

James, C. W., Anderson, G. E., Wen, L., et al. 2019, MNRAS, 489, L75

Kapadia, S. J., Caudill, S., Creighton, J. D. E., et al. 2020, CQGra, 37, 045007

Klimenko, S., \& Mitselmakher, G. 2004, CQGra, 21, S1819

Klimenko, S., Mohanty, S., Rakhmanov, M., \& Mitselmakher, G. 2005, $\mathrm{PhRvD}, 72,122002$

Klimenko, S., Mohanty, S., Rakhmanov, M., \& Mitselmakher, G. 2006, JPhCS, 32, 12

Klimenko, S., Vedovato, G., Drago, M., et al. 2011, PhRvD, 83, 102001

Klimenko, S., Vedovato, G., Drago, M., et al. 2016, PhRvD, 93, 042004

Lattimer, J., \& Schramm, D. 1976, ApJ, 210, 549

Lee, W. H., \& Ramirez-Ruiz, E. 2007, NJPh, 9, 17

Li, L.-X., \& Paczynski, B. 1998, ApJL, 507, L59

LIGO Scientific Collaboration, V. C. 2017a, GCN, 21505, 1

LIGO Scientific Collaboration, V. C. 2017b, GCN, 21513, 1

LIGO Scientific Collaboration, V. C. 2019, GCN, 24045, 1

LIGO Scientific Collaboration, V. C. 2020a, GCN, 27977, 1

LIGO Scientific Collaboration, V. C. 2020b, GCN, 27965, 1

LIGO Scientific Collaboration, V. C. 2020c, GCN, 27963, 1

LIGO Scientific Collaboration, V. C. 2020d, GCN, 27951, 1

LIGO Scientific Collaboration, V. C. 2020e, GCN, 27987, 1

LIGO Scientific Collaboration, V. C. 2020f, GCN, 27988, 1

LIGO Scientific Collaboration, V. C. 2020g, GCN, 27989, 1

LIGO Scientific Collaboration, V. C. 2020h, GCN, 27990, 1

Liu, Y., Du, Z., Chung, S. K., et al. 2012, CQGra, 29, 235018

Luan, J., Hooper, S., Wen, L., \& Chen, Y. 2012, PhRvD, 85, 102002

Macleod, D., Urban, A. L., Coughlin, S., et al. 2020, gwpy/gwpy: 2.0.2, v. v2.0.2, Zenodo, doi:10.5281/zenodo.4301851

McKinney, W. 2010, in Proc. 9th Python in Science Conf., Data Structures for Statistical Computing in Python, ed. S. van der Walt \& J. Millman, 56

Messick, C., Blackburn, K., Brady, P., et al. 2017, PhRvD, 95, 042001

Metzger, B., \& Berger, E. 2012, ApJ, 746, 48

Metzger, B., Martinez-Pinedo, G., Darbha, S., et al. 2010, MNRAS, 406, 2650

Metzger, B. D., \& Zivancev, C. 2016, MNRAS, 461, 4435

Nakar, E., \& Piran, T. 2011, Natur, 478, 82

Nicholl, M., Berger, E., Kasen, D., et al. 2017, ApJL, 848, L18

Nitz, A. H., Dal Canton, T., Davis, D., \& Reyes, S. 2018, PhRvD, 98, 024050

Nitz, A. H., Schäfer, M., \& Dal Canton, T. 2020, ApJL, 902, L29

Özel, F., \& Freire, P. 2016, ARA\&A, 54, 401

Perez, F., \& Granger, B. E. 2007, CSE, 9, 21

Sachdev, S., Magee, R., Hanna, C., et al. 2019, arXiv:1901.08580

Sachdev, S., Magee, R., Hanna, C., et al. 2020, ApJL, 905, L25

Sathyaprakash, B. S., \& Dhurandhar, S. V. 1991, PhRvD, 44, 3819

Singer, L. P., \& Price, L. R. 2016, PhRvD, 93, 024013

Solem, A. 2020, Celery, v.v4.4.2

Tohuvavohu, A., Kennea, J. A., DeLaunay, J., et al. 2020, ApJ, 900, 35

Troja, E., Rosswog, S., \& Gehrels, N. 2010, ApJ, 723, 1711

Tsang, D., Read, J. S., Hinderer, T., Piro, A. L., \& Bondarescu, R. 2012, PhRvL, 108, 011102

Urban, A. L. 2016, PhD thesis, University of Wisconsin Milwaulkee

Viets, A. D., Wade, M., Urban, A. L., et al. 2018, CQGra, 35, 095015

Zhu, X., Thrane, E., Oslowski, S., Levin, Y., \& Lasky, P. D. 2018, PhRvD, 98, 043002 